\title{
AKAP4 Gene Expression as a Candidate Breast Cancer Diagnostic Marker Detectable in Peripheral Blood
}

\author{
Motahareh Sheikhhosseini ${ }^{1}$, Mahdieh Salimi ${ }^{2, *}$ \\ ${ }^{1}$ East Tehran Department, Islamic Azad University, Tehran, Iran \\ ${ }^{2}$ Department of Medical Genetics, Institute of Medical Biotechnology, National \\ Institute of Genetic Engineering and Biotechnology (NIGEB), Tehran, Iran \\ * Corresponding author: Mahdieh Salimi, Department of Medical Genetics, Institute \\ of Medical Biotechnology, National Institute of Genetic Engineering and Biotechnology \\ (NIGEB), Tehran, Iran.E-mail: salimi@nigeb.ac.ir
}

DOI: $10.21859 / \mathrm{mci}-$ supp-06

Keywords:

AKAP4

Diagnostic Biomarker

Gene Expression

\begin{abstract}
Introduction: Breast cancer is one of the most common types of cancer which causes death among women every year. More reports on breast cancer suggest that early diagnosis is important for improving clinical outcomes and overall survival in patients with breast cancer. Cancer testis antigens (CTAs) are considered as therapeutic targets, which are promising candidates in this field. In this context, we followed the expression of AKAP4 (cancer testis antigen that expressed in breast cancer tumors) in the peripheral blood as a possible diagnostic biomarker.

Materials and Methods: To evaluate the expression of AKAP4 gene, nested real time RTPCR method with SYBR green dye was used in the blood and tissue of breast cancer patients compared with normal blood and breast tissue in the presence of an internal control in 120 samples.

Results: Our data showed that the presence of AKAP4 gene expression in breast cancer tumor tissues and its subsequent detection in peripheral blood could be considered as a possible biomarker for breast cancer diagnosis even at the early stages.

Conclusions: AKAP4 gene expression analysis in blood could be considered as a possible early diagnostic biomarker in breast cancer that is an urgent need for better management of cancer.
\end{abstract}

